University of Nebraska - Lincoln

DigitalCommons@University of Nebraska - Lincoln

Publications, Agencies and Staff of the U.S.

Department of Commerce

U.S. Department of Commerce

2012

\title{
Identification of ovarian gene expression patterns during vitellogenesis in Atlantic cod (Gadus morhua)
}

Timothy S. Breton

University of New Hampshire - Main Campus

Janet L. Anderson

University of New Hampshire - Main Campus, Janet.Anderson@unh.edu

Frederick W. Goetz

NOAA

David L. Berlinsky

University of New Hampshire - Main Campus, david.berlinsky@unh.edu

Follow this and additional works at: https://digitalcommons.unl.edu/usdeptcommercepub

Part of the Environmental Sciences Commons

Breton, Timothy S.; Anderson, Janet L.; Goetz, Frederick W.; and Berlinsky, David L., "Identification of ovarian gene expression patterns during vitellogenesis in Atlantic cod (Gadus morhua)" (2012).

Publications, Agencies and Staff of the U.S. Department of Commerce. 378.

https://digitalcommons.unl.edu/usdeptcommercepub/378

This Article is brought to you for free and open access by the U.S. Department of Commerce at DigitalCommons@University of Nebraska - Lincoln. It has been accepted for inclusion in Publications, Agencies and Staff of the U.S. Department of Commerce by an authorized administrator of DigitalCommons@University of Nebraska - Lincoln. 


\title{
Identification of ovarian gene expression patterns during vitellogenesis in Atlantic cod (Gadus morhua)
}

\author{
Timothy S. Breton ${ }^{\mathrm{a}}$, Janet L. Anderson ${ }^{\mathrm{b}}$, Frederick W. Goetz ${ }^{\mathrm{c}}$, David L. Berlinsky ${ }^{\mathrm{a}, *}$ \\ a Department of Biological Sciences, University of New Hampshire, 38 College Road, Durham, NH 03824, USA \\ ${ }^{\mathrm{b}}$ Molecular, Cellular, and Biomedical Sciences, University of New Hampshire, 46 College Road, Durham, NH 03824, USA \\ ${ }^{\mathrm{c}}$ NOAA Northwest Fisheries Science Center, Manchester Research Station, Port Orchard, WA 98366, USA
}

\section{A R T I C L E I N F O}

Article history:

Received 8 May 2012

Revised 27 August 2012

Accepted 5 September 2012

Available online 13 September 2012

\section{Keywords:}

Atlantic cod

Gene expression

Ovarian development

Vitellogenesis

Reproductive endocrinology

\begin{abstract}
A B S T R A C T
Follicular maturational competence and ovulatory competence in teleost fish refer to the ability of the ovarian follicle to undergo final oocyte maturation and ovulation, respectively, in response to gonadotropin stimulation and other external cues. Some gene products related to competence acquisition are likely synthesized during vitellogenic growth, as these follicles gain in vivo responsiveness to exogenous gonadotropin stimulation and can be induced to undergo maturation and ovulation. In Atlantic cod (Gadus morhua), gonadotropin responsiveness has been shown to be oocyte size-dependent, and only ovaries containing late-stage vitellogenic follicles can be induced to ovulate. The purpose of the present study was to compare gene expression patterns between mid (unresponsive) and late (responsive) vitellogenic ovaries to identify genes involved in gonadotropin responsiveness and the acquisition of maturational and ovulatory competencies. Representational difference analysis was conducted in two reciprocal comparisons using intact ovarian fragments and follicle wall-enriched tissues, and genes of interest were used in real time quantitative PCR to confirm differential expression. Few differences were detected in intact ovarian fragments, but type IV ice-structuring protein and gephyrin were upregulated later in development and may be involved in lipid and sulfur metabolism, respectively. Candidate gene assays for luteinizing hormone receptor and aromatase also exhibited significant upregulation during vitellogenesis. Many genes were differentially expressed in follicle wall-enriched tissues, including endocrine maturational regulators and smooth muscle genes. Overall, maturational and ovulatory competencies during vitellogenesis in Atlantic cod are associated with up- and downregulation of many genes involved in lipid metabolism, endocrine regulation, and ovulatory preparation.
\end{abstract}

(c) 2012 Elsevier Inc. All rights reserved.

\section{Introduction}

Vertebrate oogenesis is a developmental process during which primordial germ cells develop into mature, haploid ova. Early in development, oocytes become enveloped by steroidogenic follicle cells (granulosa and theca) that support their meiotically-arrested growth. The vast majority of oocyte growth occurs during

\footnotetext{
Abbreviations: MIS, maturation inducing steroid; FOM, final oocyte maturation; GVM, germinal vesicle migration; GVBD, germinal vesicle breakdown; RDA, representational difference analysis; UTR, untranslated region; QPCR, real time quantitative PCR; 20 $\beta$-HSD, 20 $\beta$-hydroxysteroid dehydrogenase; CYP19A1, aromatase; FSH-R, follicle stimulating hormone receptor; LH-R, luteinizing hormone receptor; CYP11A1, cytochrome P450 side chain cleavage; ef $1 \alpha$, eukaryotic elongation factor $1 \alpha$; $3 \beta$-HSD, $3 \beta$-hydroxysteroid dehydrogenase; AFPIV, type IV icestructuring protein; CDS, coding domain sequence; SREB1, super conserved receptor expressed in brain $1 ; 17 \beta-H S D, 17 \beta$-hydroxysteroid dehydrogenase; LH, luteinizing hormone.

* Corresponding author. Fax: +1 16038623784 .

E-mail address: david.berlinsky@unh.edu (D.L. Berlinsky).
}

vitellogenesis, as estrogen produced by the follicle cells stimulates hepatic vitellogenin synthesis and secretion into the systemic circulation. The enormous size attained by oviparous fish oocytes is primarily due to vitellogenin sequestration by receptor-mediated endocytosis and subsequent proteolytic cleavage to yolk proteins and free amino acids. Following vitellogenesis, follicles attain the ability to respond to a gonadotropin surge (follicular maturational competence, [5]) and undergo a cascade of developmental events mediated by a maturation-inducing steroid (MIS) [59]. These events include final oocyte maturation (FOM) and ovulation, and post-vitellogenic follicles must be competent to undergo both maturational and ovulatory processes to respond to the gonadotropin surge, resume meiosis, and release viable ova.

Attaining maturational and ovulatory competencies in teleost fish ovarian follicles involve orchestrated gene expression at the post-vitellogenic period, including up- and downregulation of steroidogenic enzymes during the transition from estrogen to progestin-dominated steroidogenesis [54] as well as changes in 
gonadotropin receptors, paracrine regulators, proteases [6], and inflammation-related genes [7,8]. Gene products related to maturational and ovulatory competencies, however, may also be synthesized earlier in development, as vitellogenic follicles are responsive to in vivo exogenous gonadotropin stimulation and can be induced to undergo FOM and ovulation [72]. Gonadotropin responsiveness during vitellogenesis has been shown to be oocyte size-dependent, as ovaries containing late-stage vitellogenic follicles can be induced to ovulate $[4,45]$ while smaller follicles do not respond or exhibit reduced egg viability and fertilization $[17,28]$. In Atlantic cod (Gadus morhua), which exhibits a group synchronous ovarian development, ovaries containing the largest clutch of $>675 \mu \mathrm{m}$ oocyte diameter can respond to exogenous stimulation [21] while those with only smaller oocyte populations do not.

The objective of the present study was to identify ovarian gene expression patterns during vitellogenesis in Atlantic cod that influence in vivo gonadotropin responsiveness and the acquisition of maturational and ovulatory competencies. Atlantic cod reproduction has been well studied because of its importance to commercial fisheries and aquaculture, and available resources include ultrastructural ovarian stage descriptions [34,52], seasonal reproductive steroid profiles [13], an ovarian expressed sequence tag (EST) library [23], and a recently annotated genome assembly [68]. Additionally, expression profiles for cod gonadotropins and their receptors have been assessed throughout the reproductive cycle $[50,51]$. In this study, a subtractive hybridization-based approach was used to compare gene expression patterns between mid $(<675 \mu \mathrm{m}$ maximum oocyte diameter) and late $(>675 \mu \mathrm{m})$ vitellogenic stage ovaries. Since oocytes contain abundant RNAs that likely mask rarer messages produced by the follicle cells [23], two comparisons were conducted using (1) intact ovarian fragments to identify oocyte transcripts, and (2) follicle/interstitial cell mRNA enrichments $[38,43]$. Quantitative PCR assays were developed for genes of interest to confirm differential expression, and the expression of additional candidate genes for steroidogenic enzymes and gonadotropin receptors were investigated.

\section{Methods}

\subsection{Animals and sampling}

Juvenile Atlantic cod were hatchery reared from captive broodstock (2007-2008) at Great Bay Aquaculture, LLC (Portsmouth, NH, USA) and raised in offshore net pens (University of New Hampshire Atlantic Marine Aquaculture Center). At approximately 1.5 years of age, juveniles were transported to recirculating systems (Durham, $\mathrm{NH}$, USA). Fish were raised under University of New Hampshire Institutional Animal Care and Use Committee guidelines, fed a commercial diet (Skretting, Stavanger, Norway), kept at $8-12{ }^{\circ} \mathrm{C}$, and held at a simulated natural photoperiod for 4-6 months prior to sampling. Cod entering their first reproductive season (2.0 years old, $>30 \mathrm{~cm}$ total length) were anesthetized in $50 \mathrm{mg} / \mathrm{L}$ tricaine methanesulfonate (MS-222, Argent Chemical Laboratories, Redmond, WA, USA), and ovarian development was assessed by biopsy using a $1.6 \mathrm{~mm}$ internal diameter polypropylene cannula and dissecting microscope with a stage micrometer [65]. Cod with mid $(400-600 \mu \mathrm{m})$ or late $(700-850 \mu \mathrm{m})$ vitellogenic follicles were euthanized with $200 \mathrm{mg} / \mathrm{L}$ MS-222, and their ovaries were dissected and held on ice.

Ovarian samples were collected for detailed oocyte size measurements, routine histology, and nucleic acid extractions. Samples for size measurements and histology were preserved in Ringer's solution and $10 \%$ formalin, respectively, while ovarian fragments for nucleic acid analyses were stored in RNALater (Ambion, Austin, TX, USA) and kept at $-70^{\circ} \mathrm{C}$. Additional nucleic acid samples were also processed immediately to enrich for follicle wall and interstitial cell mRNAs [38]. Briefly, ovary fragments $\left(\sim 4.0 \mathrm{~cm}^{3}\right)$ were removed from the ovarian wall and immediately deyolked by compression between two pieces of stainless steel mesh $(70 \times 70$ perforations $\left(\mathrm{cm}^{2}\right.$ ). This procedure lysed ovarian follicles and separated the extrafollicular tissue and follicle cell layers from the intracellular egg yolk. Follicle wall-enriched tissues were washed 2-3 times with ice-cold Ringer's solution and centrifuged at $5,000 \mathrm{~g}$ for $5 \mathrm{~min}$ to remove residual yolk. Tri Reagent (Sigma Aldrich, St. Louis, MO, USA) was added to each pellet (3-6 ml), and samples were homogenized using a hand-held homogenizer (Kinematica, Switzerland) prior to storage at $-70^{\circ} \mathrm{C}$.

To accurately determine oocyte size, ovarian samples in Ringer's solution were photographed with a Sedgewick Rafter counting cell with $1 \mathrm{~mm}$ squares (Aquatic Ecosystems, Apopka, FL, USA) under a dissecting microscope. Images were recorded in duplicate for each sample and analyzed using Image J software [1]. Oocyte diameters were averaged from each image (10 largest oocytes/image) to determine representative oocyte sizes. Late-stage vitellogenic follicles were also examined for evidence of FOM by clearing with ethanol:formalin:acetic acid $(6: 3: 1)$ for $1-4$ h to clarify oocyte lipid content and observe germinal vesicle migration (GVM) or breakdown (GVBD) [33]. Histological samples were preserved in $10 \%$ formalin for $24-48 \mathrm{~h}$ and processed for routine hemotoxylin and eosin staining [27]. Histological slides were examined using a compound microscope and observed for GVM, GVBD, or follicular atresia (Fig. 1).

\subsection{RNA extractions and $c D N A$ synthesis}

Intact ovarian fragments were removed from RNALater, blotted on Whatman \#1 filter paper (Whatman Inc., Sanford, ME, USA), sectioned using a razor blade $\left(\sim 0.3 \mathrm{~cm}^{3}\right)$, and added to $500 \mu$ l cold Tri Reagent. The tissue was then homogenized using microtubes and pestles (Fisher Scientific, Pittsburgh, PA, USA) and brought to $1.0 \mathrm{ml}$ with Tri Reagent. For follicle wall-enriched tissues, $500 \mu \mathrm{l}$ of processed tissue was briefly homogenized with a pestle before addition of Tri Reagent. Extractions were performed using standard phenol/chloroform procedures (Molecular Research Center, Cincinnati, OH, USA). For the intact ovarian fragment comparison, total RNA $(5 \mu \mathrm{g})$ was pooled from three individuals at mid $(474 \pm 5$, $475 \pm 9,490 \pm 10 \mu \mathrm{m})$ and late $(736 \pm 21,811 \pm 19,815 \pm 17 \mu \mathrm{m})$ vitellogenesis. For the follicle wall-enriched comparison, new samples from mid vitellogenesis $(485 \pm 9,508 \pm 9,587 \pm 10 \mu \mathrm{m}$ ) were used while late vitellogenic processed tissues were available from the same individuals as those used for intact preparations. The SMARTer cDNA synthesis kit (Clontech, Mountain View, CA, USA) was used to amplify full-length cDNAs prior to subtractive hybridization procedures.

\subsection{Subtractive hybridization and analysis}

Representational difference analysis (RDA; [26,40]) was conducted in reciprocal experiments for intact ovarian fragment and follicle wall-enriched comparisons using an optimized protocol by Pastorian et al. [58] and modified by Anderson et al. [2]. Briefly, cDNA populations $(5 \mu \mathrm{g})$ from mid and late vitellogenic tissues were digested with DpnII restriction enzyme (NEBioLabs, Ipswich, MA, USA) and used in driver and tester preparations [58]. Subtractive hybridization was performed at a 1:10 tester:driver ratio over $25 \mathrm{~h}$ and used in PCR to differentially amplify expressed cDNA fragments. PCR products were extracted using phenol/chloroform and used in two additional rounds of subtractive hybridization and PCR. For each successive round, new primer sets were used [61] and tester:driver ratios were reduced to $1: 100$ and 1:5000, respectively. 

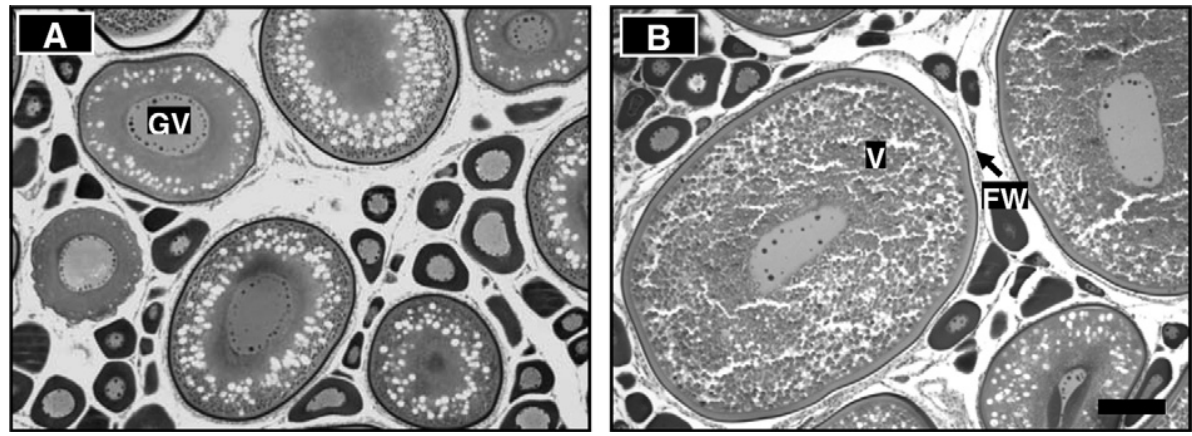

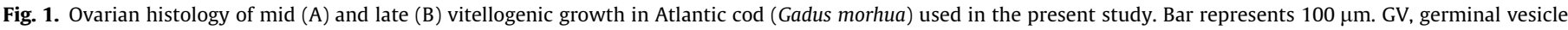
(nucleus); V, yolk proteins derived from vitellogenins; FW, follicle wall (theca and granulosa cells).

PCR products following three RDA rounds were ligated into pBluescript II SK + phagemid vectors (Agilent Technologies, Santa Clara, CA, USA) and transformed into chemically competent Escherichia coli XL1 MRF' cells (Agilent Technologies). Subtracted cDNA libraries were constructed from plated colonies, and approximately 400-500 clones per library were randomly sequenced using the dideoxy chain termination method with Big Dye Terminator (Applied Biosystems, Foster City, CA) and T7 primer with standard techniques. The reactions were precipitated and resuspended in Hi-Di Formamide with EDTA (Applied Biosystems) and run on an ABI Prism 3730 automated sequencer (Applied Biosystems). Sequence chromatograms were trimmed for quality, vector-screened, and analyzed locally using: 1 ) blastx against NCBI nonredundant (nr) protein database, 2) blastn against NCBI nucleotide (nt) database, and 3) blastn against NCBI G. morhua EST database. Unknown transcripts with significant similarity to G. morhua ESTs were further evaluated for possible homology to known protein-coding genes using the UniGene database (NCBI, Bethesda, MD, USA). The Atlantic cod first draft genome assembly (gadMor1) available on Ensembl software (European Bioinformatics Institute, Cambridge, UK) was used to identify selected transcripts as likely 3' untranslated regions (UTRs) of known genes.

\subsection{Real time quantitative PCR (QPCR)}

To confirm differential expression, primer sets were developed (NCBI Primer-Blast) from sequences of interest and used in QPCR (Table 1). All primer sets were designed to amplify $80-150 \mathrm{bp}$ fragments and verified for target specificity using the NCBI G. morhua nucleotide database. Previously developed assays $[49,50]$ for candidate genes involved in ovarian development were also used,

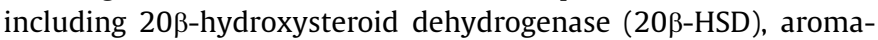
tase (CYP19A1), follicle-stimulating hormone receptor (FSH-R), and luteinizing hormone receptor (LH-R). QPCR primers for cytochrome P450 side chain cleavage enzyme (CYP11A1) were developed from an Atlantic cod cDNA sequence (GenBank Accession \# AY706102.1; [23]). To accurately compare relative gene expression changes with previous research [50], candidate gene assays were conducted using intact ovarian fragments.

RNA extractions for QPCR assays were performed as described in section 2.2 using the same individual samples from each comparison ( $n=3$ per stage), and mRNA was isolated using the MicroPoly(A) Purist kit (Ambion, Austin, TX, USA) and quantified using an ND 1000 NanoDrop spectrophotometer (Thermo Scientific, Wilmington, DE, USA). cDNA synthesis was performed using $100 \mathrm{ng}$ of mRNA and SuperScript III reverse transcriptase (Invitrogen, Carlsbad, CA) prior to relative quantification SyberGreenER QPCR (Invitrogen).

QPCR assays were performed using an ABI 7500 Fast System (Applied Biosystems) with $75 \mathrm{nM}$ to $1.2 \mu \mathrm{M}$ primer concentrations under standard cycling conditions: $50{ }^{\circ} \mathrm{C}$ for $2 \mathrm{~min}$, $95^{\circ} \mathrm{C}$ for
$10 \mathrm{~min}, 40$ cycles of $95^{\circ} \mathrm{C}$ for $15 \mathrm{~s}$ and $60^{\circ} \mathrm{C}$ for $1 \mathrm{~min}$, followed by dissociation curve analysis. Samples and negative controls were performed in duplicate $(1 / 10$ or $1 / 100$ diluted), and triplicate relative standard curves $(1 / 5-1 / 10,000)$ were generated from dilutions of pooled cDNA from six individuals. Optimized assays consisted of five point linear standard curves with approximately 90-110\% PCR efficiency, no contamination $<10 \mathrm{Ct}$ from samples [44], and presence of a single peak in dissociation curve analysis.

Since reference genes ( $18 \mathrm{~S}$ rRNA, $\beta$-actin, ef $1 \alpha$ ) used previously exhibit seasonal variation in Atlantic cod ovarian follicles, and do not meet stability criteria [49], results were analyzed using the relative standard curve method (Applied Biosystems User Bulletin \#2) and not normalized to a reference gene. Rather, mRNA input was standardized using the methods of Campbell et al. [10], and each assay was contained within one 96-well plate. One-way ANOVAs were performed (JMP 8.0, SAS Institute, Cary, NC, USA) using log-transformed relative sample concentrations to identify significant differences $(\alpha<0.05)$ in gene expression between stages. The lowest individual gene expression value was arbitrarily set to 1 , to enhance data presentation [44].

\subsection{Follicle nucleic acid assessments}

To assess RNA changes during vitellogenesis on a per follicle basis, follicle numbers, RNA content, and mRNA content per tissue mass were determined from the same individuals used in each comparison. For intact ovarian fragments ( $50 \mathrm{mg})$, vitellogenic follicles were counted using a dissecting microscope and used in RNA and mRNA extractions. For follicle wall-enriched tissues, $500 \mu \mathrm{l}$ homogenized sample was centrifuged at $12,000 \mathrm{x} g$ for $5 \mathrm{~min}$, and $10 \mathrm{mg}$ pellet $+300 \mu \mathrm{l}$ supernatant was used in RNA extractions, DNA extractions (Molecular Research Center), and mRNA isolations. DNA content was used as a relative estimate of cell number per tissue mass, as whole follicles could not be counted following the enrichment procedure. One-way ANOVAs were performed (JMP 8.0) to identify significant differences in nucleic acid content between stages. Percent data were arcsine transformed prior to statistical analyses.

\section{Results}

\subsection{Intact ovarian fragment comparison}

The cDNA library putatively enriched for transcripts upregulated in late vitellogenesis contained approximately 400 sequences (>60 bp) with a mean size of $300 \mathrm{bp}$. Most sequences (96\%) exhibited significant similarity (e value $<1.0^{-7}$ ) to annotated genes and $4 \%$ exhibited similarity to genes of unknown function (e.g., hypothetical proteins). A single transcript with similarity to syntaxin 2 comprised approximately $35.8 \%$ of all sequences. The cDNA library 
Table 1

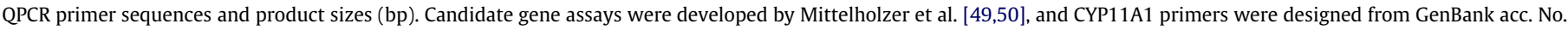
AY706102.1.

\begin{tabular}{|c|c|c|c|}
\hline Gene name/function & $5^{\prime}$-Forward primer-3' & $5^{\prime}$-Reverse primer- $3^{\prime}$ & Product size (bp) \\
\hline \multicolumn{4}{|l|}{ Intact ovarian fragment comparison } \\
\hline \multicolumn{4}{|l|}{ RDA-identified genes } \\
\hline $3 \beta$-HSD- $\Delta 8, \Delta 7$-isomerase & GCCGACCAGAGCTTCCTATCCCA & AAGGGTCCCCACAGCCAGGC & 122 \\
\hline Ceramide synthase 5 & TACCGGTCGTGGTGGCTGCT & TGGCGATGCGAGCGATGAGG & 88 \\
\hline Cyclin B2 & GGCCGGTAGTGCACCATGGC & TCAGGAGAGCCTCAAAGGCTGCA & 106 \\
\hline Gephyrin & CCGCTGCATCCTGACCTGGC & CGCATGCTCATCAGCCGGCT & 90 \\
\hline Hypothetical protein (wu:fb21f05) & TGAGCCGGCCGCCAGTCTAT & GCCGCCATGCTGGAGGTGAG & 104 \\
\hline Hypothetical protein (zgc:112095) & CGGCTCGCTCGCTCCTTCAG & GCTGCAGGCTCCCCAGAGGA & 110 \\
\hline Pyridoxal phosphate phosphatase & GCCCTTGTGCGCCGAGATGA & CCTGACGGCATTCGCGTCGT & 87 \\
\hline RuvB-like1 & CAGCAGGTCCAGCGGGATGC & GTGTTCGCCTCCAACCGGGG & 84 \\
\hline Secretogranin III & CGGCGAAGTCCGGATTGCCC & CCATCACGCCCGAGGAGGGA & 108 \\
\hline Syntaxin 2 & TCGAGTCCCGGCACCATGACA & GGTGTCTACCAGCACGGCGG & 92 \\
\hline Type IV ice-structuring protein & TCGAGCTGGGTCCTCCCGTC & TGCCGTAGAGCAGAGCCCAG & 136 \\
\hline Zona pellucida glycoprotein C & GGGTGTCCTTGGTCACCGTCC & TCTCCTGCGACTCGGCAGCT & 110 \\
\hline \multicolumn{4}{|l|}{ Candidate genes } \\
\hline $20 \beta-H S D$ & GGTCAACAATGCTGGGATAGC & GCCTGCACGGCAAACG & 60 \\
\hline FSH receptor (FSH-R) & CACGCCAACCTCACCTATCAC & TGAACAGATGGAGTCCCACTTG & 81 \\
\hline LH receptor $(\mathrm{LH}-\mathrm{R})$ & GCCACTGTTGTGCCTTCCA & GAGCCTTGTGAGGTTCTTTAATGC & 62 \\
\hline P450 aromatase (CYP19A1) & ACAACAACAAGTACGGCAGCAT & GTAGAGGAGCTGCTGAGGATGAG & 76 \\
\hline P450 side chain cleavage (CYP11A1) & AGGGCTGTTTGCGATGGGCC & GGCCGAATCCGAAGCCCAGG & 119 \\
\hline \multicolumn{4}{|l|}{ Follicle wall-enriched comparison } \\
\hline \multicolumn{4}{|l|}{ RDA-identified genes } \\
\hline $17 \beta$-HSD type 1 & GGAGCGACTGCTGGGCTGTG & TGTCCCGGGCCTCCAGGATG & 101 \\
\hline$\alpha$-Actinin-4 & CGCCTCСTCСTCCTCCCTGG & TACCGGCGGACTCCATGCCA & 131 \\
\hline Claudin29a & TCTCAGACGTAGGCCCCGCC & CGCTCTGCTCTGCTGCCAGT & 102 \\
\hline Creatine kinase & CGGCGGGTACAAGCCCACAG & GTGCGCACCCTGGAGCTCAG & 108 \\
\hline Cysteine rich protein 1 & CTTTGCCCCAGCTGCCCACC & CACACGTCAGAGCCCGGCAG & 81 \\
\hline Myosin-11 CDS & AACTCAGGCGTGGCACCGAG & CTGAGGCGTCGTCCACCACG & 90 \\
\hline Myosin-11 3' UTR & GCGGACACATCAGTACACGACTGG & GTCGGTCCACCATGTCGGCC & 128 \\
\hline Oocyte-specific F-box protein & CAGGGGCAGCGAGGGAGACT & TGCTGGGACTCGGAGCCACT & 108 \\
\hline Serotonin receptor $1 \mathrm{E}$ & AGCAGGGGGTTGACCAGGGA & GCTGGTGGGGCTGAGGCTTG & 93 \\
\hline SREB1 & AGACGCACCCGGAGCTCCTT & CAGGTGTACCTCACCGCCGC & 98 \\
\hline Transgelin CDS & CTGCCCAGAGCGGAGAGGGT & CCGCCGAGAAGTACGGCGTC & 106 \\
\hline Transgelin 3' UTR & GCGCCACGGCCATCACATGT & GGGGTCCTCTGTGTGTCCTGCT & 94 \\
\hline Tropomyosin 1 alpha chain & CTTCTCGGCCTGGGCCTCCA & GCACAGAGGAGCGCGCTGAG & 107 \\
\hline Unknown transcript 1 & TGGGATGCCGCCGATTGGTT & CGCATCAGCTGGCTTCAGGGG & 85 \\
\hline Unknown transcript 2 & ACCGCTGGACCTGGACGTCA & TCGGACATGTGGAGTCGGACATG & 92 \\
\hline
\end{tabular}

putatively enriched for transcripts upregulated in mid vitellogenesis contained approximately 450 sequences with a mean size of $331 \mathrm{bp}$. Most sequences (76\%) exhibited similarity to genes of known identity, while $24 \%$ were identified as hypothetical proteins. An abundant transcript for one hypothetical protein (zgc:112095) comprised $18.7 \%$ of all clones. Sequences from intact follicle RDA libraries were submitted to the NCBI GenBank EST database (JK752908 - JK753859). From these libraries, 12 transcripts of interest were selected for further assessment by QPCR (Table 2).

Most genes assessed by QPCR within intact ovarian fragments were not differentially expressed and exhibited equal expression between vitellogenic stages, including syntaxin 2 , ceramide synthase 5 , cyclin B2, hypothetical proteins, zona pellucida glycoprotein C, $3 \beta$-HSD $\Delta 8, \Delta 7$-isomerase, ruvB-like1, and pyridoxal phosphate phosphatase $(p>0.10)$. Significant upregulation during vitellogenesis, however, was detected at two genes (Fig. 2A). Type IV ice-structuring protein (AFPIV) and gephyrin were upregulated at late vitellogenesis approximately 180 and 2-fold, respectively. Secretogranin III exhibited some upregulation, but expression was highly variable among individuals and not significant $(p=0.0804)$. Two candidate genes also exhibited differential expression between vitellogenic stages (Fig. 2B). LH-R and aromatase (CYP19A1) were significantly upregulated 12 and 4-fold, respectively, while CYP11A1 expression was variable and not significantly different between stages $(p=0.0881)$. FSH-R and $20 \beta$-HSD were also equally expressed between mid and late vitellogenic stages $(p>0.10)$.
Intact ovarian fragments exhibited approximately equal nucleic acid content during vitellogenesis (Table 3 ). Although mid vitellogenic tissues contained twice the follicle number per tissue mass, total RNA and mRNA content per follicle were equal between stages. In addition, RNA composition was similar, as percent mRNA did not significantly differ during vitellogenesis.

\subsection{Follicle wall-enriched comparison}

The cDNA library enriched for follicle wall transcripts upregulated in late vitellogenesis contained approximately 530 sequences with a mean size of $283 \mathrm{bp}$. Many sequences (45\% of all clones) exhibited no significant similarity to genes in the blastx or blastn database. Most sequences, however, comprised two distinct cDNA fragments identified by UniGene and whole genome assembly as 3' UTRs of known genes myosin-11 and transgelin. Other cDNA fragments corresponding to each gene's coding domain sequences (CDS) were also identified (Table 4).

The library enriched for follicle wall transcripts upregulated in mid vitellogenesis contained approximately 375 sequences with a mean size of $248 \mathrm{bp}$. Most sequences (64\%) showed significant similarity to genes of known function, while $28 \%$ were identified as one unknown transcript with no similarity to the G. morhua EST database (unknown transcript 1). Unknown transcript 2 also exhibited no similarity to known genes and was present in low numbers within the library (3\%). Most genes identified in the intact ovarian fragment comparison were either not present in follicle 
Table 2

Intact ovarian fragment comparison genes of interest identified by RDA and further assessed in QPCR

\begin{tabular}{|c|c|c|c|c|c|c|}
\hline Gene name/function & EST & Size (bp) & Blastx score & Species most similar to & Accession number of similar protein & Percent library (\%) \\
\hline \multicolumn{7}{|l|}{ Late vitellogenic cDNA library } \\
\hline Syntaxin 2 & JK752925 & 311 & $1.0 \mathrm{E}-16$ & Coturnix coturnix & BAC00814.1 & 35.8 \\
\hline Type-IV ice-structuring protein & JK752931 & 238 & $6.0 \mathrm{E}-24$ & Gadus morhua & Q56TU0.1 & 9.5 \\
\hline Ceramide synthase 5 & JK753085 & 255 & $3.0 \mathrm{E}-15$ & Homo sapiens & NP_671723.1 & 5.0 \\
\hline Cyclin B2 & JK753043 & 340 & $1.0 \mathrm{E}-36$ & Oncorhynchus mykiss & NP_001118131.1 & 3.8 \\
\hline Gephyrin & JK753138 & 412 & $1.0 \mathrm{E}-68$ & Danio rerio & XP_002663725.1 & 2.5 \\
\hline Hypothetical protein (wu:fb21f05) & JK753034 & 310 & $5.0 \mathrm{E}-22$ & Danio rerio & XP_001340467.2 & 2.0 \\
\hline Zona pellucida glycoprotein $\mathrm{C}$ & JK752929 & 281 & $1.0 \mathrm{E}-15$ & Cynoglossus semilaevis & ABY81291.1 & 1.3 \\
\hline $3 \beta$-HSD- $\Delta 8, \Delta 7$-isomerase & JK753128 & 188 & $1.0 \mathrm{E}-25$ & Anoplopoma fimbria & ACQ57982.1 & 0.2 \\
\hline Secretogranin III & JK753049 & 283 & $7.0 \mathrm{E}-31$ & Danio rerio & CAF99835.1 & 0.2 \\
\hline \multicolumn{7}{|l|}{ Mid vitellogenic $c D N A$ library } \\
\hline Hypothetical protein (zgc:112095) & JK753529 & 258 & $5.0 \mathrm{E}-11$ & Danio rerio & CAN88628.1 & 18.7 \\
\hline RuvB-like1 & JK753466 & 250 & $2.0 \mathrm{E}-38$ & Salmo salar & NP_001133819.1 & 4.7 \\
\hline Pyridoxal phosphate phosphatase & JK753606 & 410 & $7.0 \mathrm{E}-36$ & Salmo salar & ACI33038.1 & 4.5 \\
\hline
\end{tabular}

A

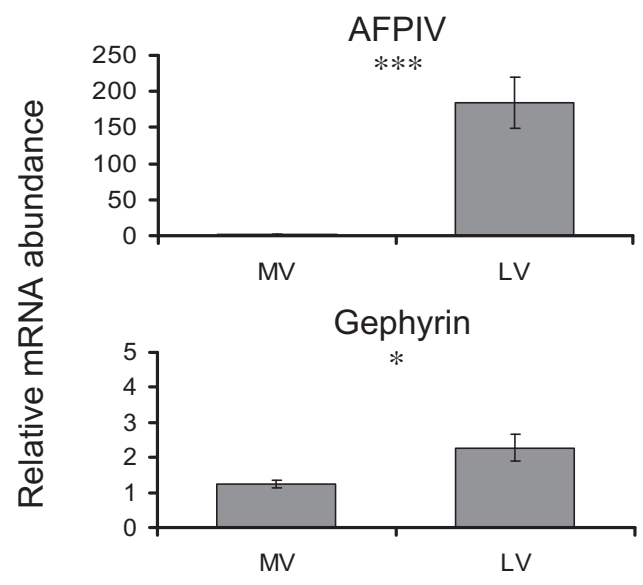

B

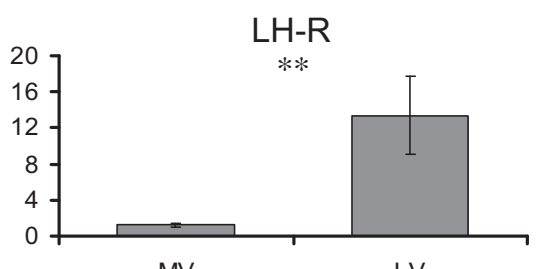

LV

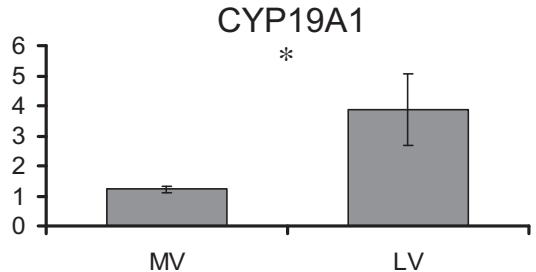

\section{Ovarian stage}

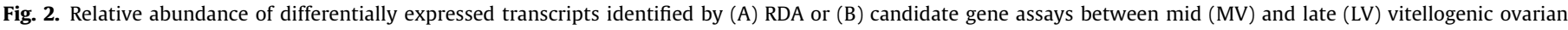
fragments. Each bar represents the mean \pm standard error with ${ }^{*},{ }^{* *}$, and ${ }^{* * *}$ indicating significance at $p<0.05,0.01$, and 0.001 levels, respectively.

Table 3

Nucleic acid assessments of $50 \mathrm{mg}$ intact ovarian fragments at mid (MV) and late (LV) vitellogenesis. Different letters denote significant differences.

\begin{tabular}{lcr}
\hline Parameter & \multicolumn{2}{l}{ Ovarian stage } \\
\cline { 2 - 3 } & MV & \multicolumn{1}{c}{ LV } \\
\hline Vitellogenic follicle count & $1135.9 \pm 70.6^{\mathrm{a}}$ & $452.2 \pm 18.2^{\mathrm{b}}$ \\
$\mu \mathrm{g}$ total RNA/follicle & $0.43 \pm 0.07$ & $0.51 \pm 0.04$ \\
ng mRNA/follicle & $16.47 \pm 1.32$ & $24.19 \pm 2.68$ \\
ng mRNA/ $\mu$ g total RNA & $41.45 \pm 8.75$ & $48.07 \pm 5.05$ \\
$\%$ mRNA & $4.15 \pm 0.86$ & $4.81 \pm 0.51$ \\
\hline
\end{tabular}

wall-enriched libraries (ceramide synthase 5, hypothetical proteins, etc.) or present only in few numbers (syntaxin 2, cyclin B2). Sequences from follicle wall-enriched RDA libraries were submitted to the NCBI GenBank EST database (JK753860 - JK754868). From these libraries, 15 transcripts of interest were identified and used in QPCR (Table 4).

Most selected transcripts ( 9 out of 15) identified in the follicle wall-enriched comparison and assessed by QPCR exhibited differential expression. Sequences corresponding to transgelin CDS and $3^{\prime}$ UTR were significantly upregulated approximately 200 fold at late vitellogenesis (Fig. 3). Serotonin receptor 1E and $\alpha$-actinin-4 were also significantly upregulated approximately 12 and 80 -fold, respectively. Super conserved receptor expressed in brain 1
(SREB1) was downregulated during vitellogenesis 5-fold. Sequences corresponding to cysteine rich protein 1 , tropomyosin 1 alpha chain, and myosin-11 CDS and $3^{\prime}$ UTR were expressed in all late vitellogenic tissues but not detected earlier in development (data not shown). Unknown transcript 1 was highly expressed in mid vitellogenic tissues (100 fold higher overall) but also extremely variable, and the comparison between stages was not significant $(p=0.0771)$. 17 $\beta$-HSD type 1 expression was overall slightly decreased at late vitellogenesis but also not significantly different between stages ( $p=0.0533$ ). Oocyte-specific $\mathrm{F}$ box protein, creatine kinase, claudin29a, and unknown transcript 2 exhibited equal expression during vitellogenesis $(p>0.10)$.

Follicle-wall enriched tissues exhibited significant nucleic acid changes during vitellogenesis (Table 5). DNA and RNA content were approximately 70 and 13 times greater in mid vitellogenic samples, respectively. Percent mRNA also significantly decreased during vitellogenesis.

\section{Discussion}

\subsection{Follicle nucleic acid composition}

Many maternal RNAs, which are synthesized early in follicular growth, accumulate within the developing oocyte $[67,71]$ and are 
Table 4

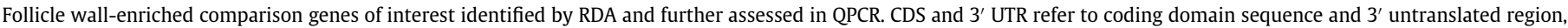
respectively.

\begin{tabular}{|c|c|c|c|c|c|c|}
\hline Gene name/function & EST & Size (bp) & Blastx score & Species most similar to & Accession number of similar protein & Percent library (\%) \\
\hline \multicolumn{7}{|l|}{ Late vitellogenic cDNA library } \\
\hline Transgelin $3^{\prime}$ UTR & JK753925 & 289 & - & - & - & 28.2 \\
\hline Myosin-11 $3^{\prime}$ UTR & JK753928 & 227 & - & - & - & 14.3 \\
\hline Myosin-11 CDS & JK753992 & 356 & $5.0 \mathrm{E}-24$ & Salmo salar & ACN58605.1 & 10.7 \\
\hline Transgelin CDS & JK754113 & 235 & $6.0 \mathrm{E}-31$ & Esox lucius & AC014008.1 & 8.5 \\
\hline Cysteine rich protein 1 & JK753971 & 221 & $3.0 \mathrm{E}-28$ & Osmerus mordax & ACO09474.1 & 7.5 \\
\hline Tropomyosin 1 alpha chain & JK753983 & 236 & $8.0 \mathrm{E}-26$ & Salmo salar & ACI34179.1 & 5.3 \\
\hline$\alpha$-actinin-4 & JK754345 & 327 & $1.0 \mathrm{E}-55$ & Rachycentron canadum & ADV76249.1 & 4.5 \\
\hline Serotonin receptor $1 \mathrm{E}$ & JK754097 & 426 & $2.0 \mathrm{E}-16$ & Zonotrichia albicollis & ADK26830.1 & 1.3 \\
\hline Oocyte-specific F-box protein & JK754001 & 327 & $4.0 \mathrm{E}-41$ & Oncorhynchus mykiss & ADN95180.1 & 0.6 \\
\hline Creatine kinase, testis isozyme & JK753949 & 424 & $9.0 \mathrm{E}-67$ & Esox lucius & AC013522.1 & 0.2 \\
\hline Claudin29a & JK754199 & 156 & $5.0 \mathrm{E}-07$ & Takifugu rubripes & AAT64062.1 & 0.2 \\
\hline \multicolumn{7}{|l|}{ Mid vitellogenic cDNA library } \\
\hline Unknown transcript 1 & JK754749 & 253 & - & - & - & 26.9 \\
\hline Unknown transcript 2 & JK754572 & 274 & - & - & - & 2.7 \\
\hline SREB1 & JK754505 & 241 & $1.0 \mathrm{E}-28$ & Danio rerio & NP_001107906.1 & 0.3 \\
\hline $17 \beta$-HSD type 1 & JK754496 & 261 & $7.0 \mathrm{E}-33$ & Oreochromis niloticus & AAV74182.1 & 0.3 \\
\hline
\end{tabular}

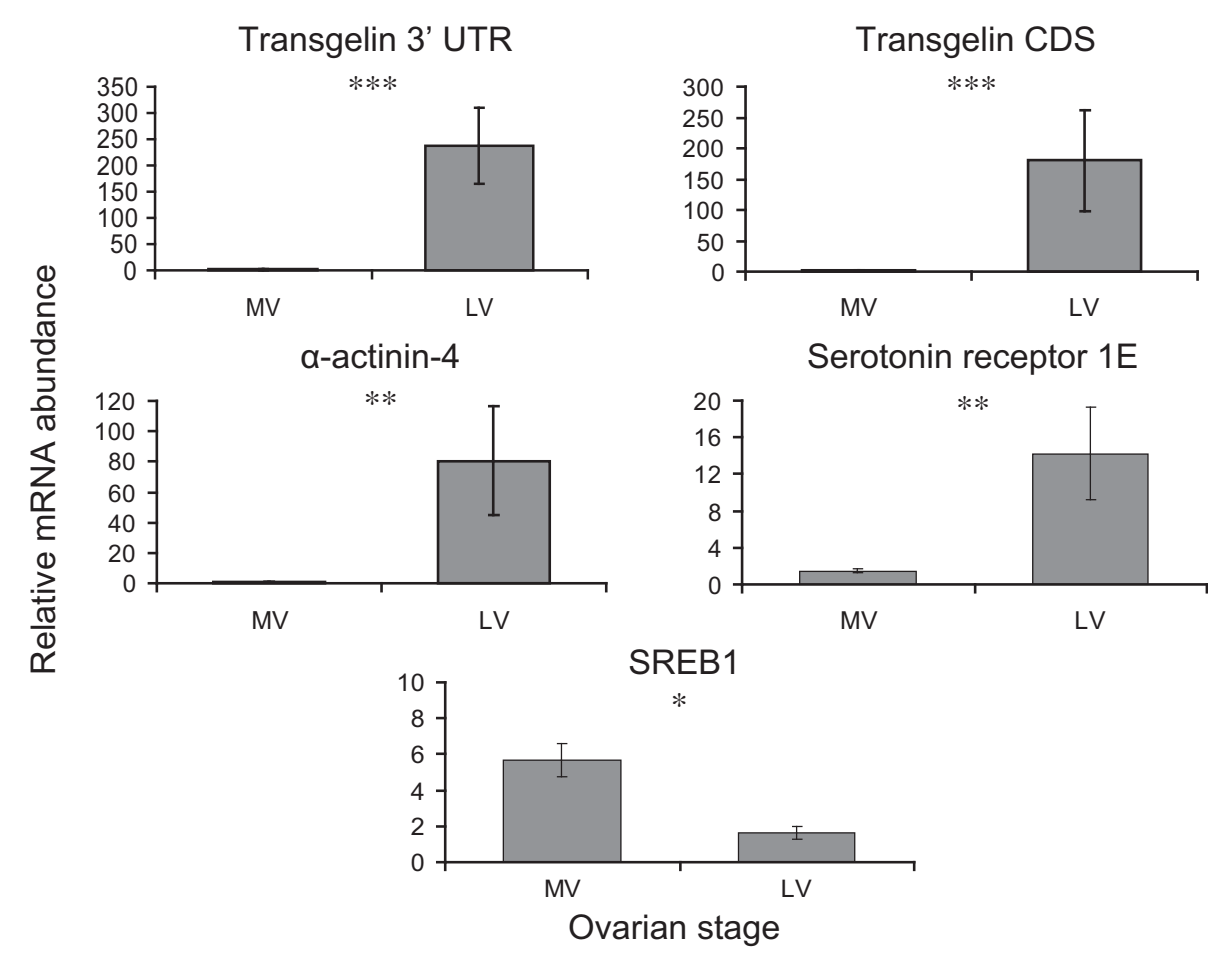

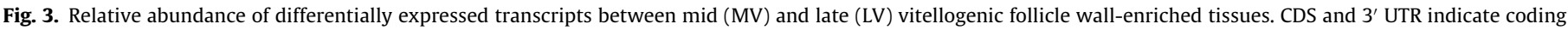

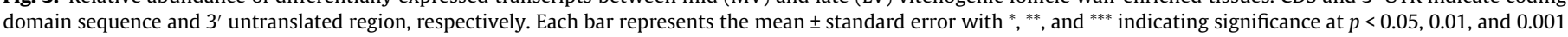
levels, respectively.

stored until FOM or embryogenesis. In the present study, many abundant, non-differentially expressed transcripts were identified in intact ovarian fragments that may represent false positives of the subtractive hybridization method [62] and are likely stored messages in oocytes. For example, cyclin B was equally expressed between stages and is not translated until FOM to regulate many nuclear and cytoplasmic changes, including GVBD and spindle formation [54]. Similarly, syntaxin 2 (also known as epimorphin [60]) may not function until fertilization $[12,29]$ or embryonic development [57]. These abundant stored messages in vitellogenic oocytes greatly impacted our ability to identify differential gene expression patterns, as intact mid and late vitellogenic tissues exhibited overall equal RNA content despite dramatic follicle size differences.

In contrast, considerably fewer stored messages were identified in the follicle wall-enriched comparison, and enriched tissues
Table 5

Nucleic acid assessments of follicle wall-enriched tissue for mid (MV) and late (LV) vitellogenic stages. Different letters denote significant differences.

\begin{tabular}{lcc}
\hline Parameter & Ovarian stage \\
\cline { 2 - 3 } & MV & LV \\
\hline$\mu$ g DNA & $22.3 \pm 5.8^{\mathrm{a}}$ & $0.3 \pm 0.2^{\mathrm{b}}$ \\
$\mu \mathrm{g}$ total RNA & $481.3 \pm 49.3^{\mathrm{a}}$ & $36.8 \pm 13.0^{\mathrm{b}}$ \\
ng mRNA/ug total RNA & $42.8 \pm 2.0^{\mathrm{a}}$ & $27.6 \pm 4.4^{\mathrm{b}}$ \\
$\%$ mRNA & $4.3 \pm 1.0^{\mathrm{a}}$ & $2.8 \pm 0.4^{\mathrm{b}}$ \\
\hline
\end{tabular}

exhibited dramatic differences in nucleic acid content and tissue composition. For example, late vitellogenic tissue enrichments exhibited reduced DNA content, which may indicate greater 
quantities of acellular material such as zona pellucida proteins. Late vitellogenic follicle cells, unlike mid vitellogenic samples, may only be moderately enriched compared to the zona pellucida, since oocyte volume increases dramatically during vitellogenesis in relation to surface area. Differences in RNA content, however, were less dramatic between mid and late vitellogenic samples and indicated that considerable changes in RNA expression on a per cell basis likely occur at the follicle wall.

Gene expression changes in teleost ovaries are often difficult to interpret, as many cell types contribute to the total mRNA pool, and group synchronous strategies for ovarian development, as in Atlantic cod, result in heterogeneous populations of oocyte growth stages. Gene expression patterns analyzed using ovarian fragments, therefore, represent the sum of all transcripts present in existing follicles [31,63], and only individual follicle isolations can elucidate expression changes on a per follicle basis in group synchronous species. Despite these factors, ovarian fragment analyses provide insight into gene expression changes at the ovary level, and the comparisons used in the present study identified several up- and downregulated genes during vitellogenesis that may influence in vivo responsiveness to gonadotropins and maturational and ovulatory competencies.

\subsection{Lipid metabolism}

Type IV ice-structuring protein (AFPIV) was significantly upregulated during vitellogenesis in intact ovarian fragments. AFPIV was first identified as an antifreeze protein in teleost blood plasma [14,15], and abundant expression was detected during carp embryogenesis [41] and in an Atlantic cod ovary EST library [23]. Prior research in cod, however, has found no antifreeze activity in eggs and larvae [69], and AFPIV function as an antifreeze protein is unclear, as it only renders low thermal hysteresis [22] and is structurally similar to apolipoproteins (lipid-binding proteins) from many fish species. While oocyte lipid accumulation occurs during vitellogenic growth [42], lipid transportation and processing may also be critical later in development, as several genes related to lipid metabolism are upregulated at late vitellogenesis or FOM in rainbow trout, including apolipoprotein $C[7,9]$ and an oocyte-specific oxysterol binding protein [61]. The antifreeze activity of AFPIV may just be coincidental [39], and the true function may involve lipid binding and metabolism in the egg.

\subsection{Sulfur metabolism}

Late vitellogenic tissues exhibited small, but significant, upregulation (2-fold) of gephyrin, which is a multifunctional protein associated with synaptic receptor clustering and sulfur metabolism $[19,20]$. Gephyrin is abundant in many non-neuronal tissues for molybdenum cofactor biosynthesis, which is required for sulfite oxidation in heterotropic organisms [20]. Gephyrin upregulation within vitellogenic oocytes is likely related to sulfur metabolism either within the oocyte or as stored maternal messages for embryonic development.

\subsection{Steroidogenesis}

Aromatase was significantly elevated (4-fold) during vitellogenesis, which is consistent with peak expression late in vitellogenic growth in other teleost species [36,63]. Aromatase is critical for $17 \beta$-estradiol production in granulosa cells to regulate vitellogenin synthesis in the liver [66], and expression is only drastically reduced at the post-vitellogenic period [7,36]. Other gene expression changes related to steroidogenesis also occur later in development $[6,8,24]$, as the follicle undergoes a steroidogenic shift at FOM [54]. The overall stable expression levels observed in other steroido- genic enzymes in the present study, therefore, are consistent with vitellogenic growth prior to maturation.

\subsection{Endocrine maturational regulators}

Vitellogenesis termination and FOM induction are associated with several endocrine events, including an increase in plasma LH and high LH-R expression [8,32]. In the present study, late vitellogenic ovarian tissues exhibited significant LH-R upregulation that is consistent with a role in preparing the follicle to receive the gonadotropin signal. Prior research in cod indicated that the highest LH-R expression during the reproductive season occurred at spawning (15-fold upregulation) [50], and interestingly, these relative changes were also similar to levels in this study (12-fold), which suggest that the late vitellogenic period is responsible for most of the increase in LH-R during ovarian development.

Serotonin (5-hydroxytryptamine) receptor $1 \mathrm{E}$ was also upregulated 14-fold at late vitellogenic follicle wall-enriched tissues. Serotonin can inhibit GVBD in fish oocytes via intracellular increases in cAMP [11], however, class 1 receptors are associated with cAMP decreases and little is known concerning receptor $1 \mathrm{E}$ function [37]. Although a complex serotonergic network exists in mammalian follicle cells to regulate steroid production and maturation [16], more information is needed to understand the role of serotonin in regulating steroidogenesis and FOM in fish.

Super conserved receptor expressed in brain 1 (SREB1) was also downregulated 5-fold during vitellogenesis and is a member of an evolutionarily conserved, amine-like $G$ protein-coupled receptor family that is highly expressed in the mammalian brain $[46,47,55]$ and reproductive organs [47]. These receptors have received little attention in vertebrate reproduction, however, and endogenous ligands for these receptors remain unknown [48]. To our knowledge, the present study is the first to demonstrate differential expression of an SREB during ovarian development and may suggest a regulatory role in the follicle wall or interstitial cells that decreases as the vitellogenic follicle approaches maturation.

\subsection{Smooth muscle gene upregulation}

Most genes identified in the follicle wall-enriched comparison were associated with smooth muscle contractile machinery and cytoskeletal organization. For example, $\alpha$-actinin- 4 is involved in cell shape and motility processes [56], while cysteine rich protein 1 influences cell morphology pathways during embryonic development [53] and may be involved in smooth muscle contractile machinery organization [25]. Similarly, transgelin is an early marker of smooth muscle differentiation, but its exact functions are unknown [3]. Transgelin has previously been detected in the fathead minnow ovary, where it was highly upregulated in vitellogenic and mature follicles [70]. These results suggest that smooth muscle genes are upregulated in the late vitellogenic follicle wall and may function in ovulatory competency for follicular rupture during ovulation. Although the presence and function of smooth muscle during ovulation has been controversial for decades [18], some evidence has existed for its role in teleost follicles $[30,64]$, and mammalian theca cells can contract during follicular rupture [35]. While ovulation in fish has been described as an inflammatory-like reaction $[8,42]$, other processes such as smooth muscle contractions may cooperate with these factors, and further research is needed to investigate the role of follicular smooth muscle.

\section{Conclusions}

This study provides novel information on ovarian gene expression patterns during vitellogenesis that may influence in vivo 
gonadotropin responsiveness and acquisition of both maturational and ovulatory competencies. Although intact ovarian fragments exhibited few differences and stable expression levels during this growth period, dramatic differences in nucleic acid composition and gene expression were evident in follicle wall-enriched tissues. While the subtractive hybridization-based approach identified many gene expression patterns related to lipid metabolism, endocrine regulation of ovarian development, and smooth muscle production, further research is needed to localize gene expression to specific cell types and confirm potential functions in relation to maturational and ovulatory competencies. In addition, the recent availability of the Atlantic cod genome will enable comprehensive, whole transcriptome sequencing approaches to further identify rare gene expression patterns in the ovarian mRNA pool.

\section{Acknowledgments}

Funding was provided by New Hampshire Sea Grant and the University of New Hampshire Marine Program. The authors thank Abigail Walker and Heidi Colburn for assistance with animal husbandry and sampling. We also thank Crystal Simchick and Giles Goetz from University of Wisconsin Milwaukee for contributions in library sequencing and Dr. Wayne Decatur from the University of New Hampshire Center for Molecular and Comparative Endocrinology for invaluable assistance with Atlantic cod genomic analyses.

\section{References}

[1] M.D. Abramoff, P.J. Magelhaes, S.J. Ram, Image processing with Image J, Biophotonics Int. 11 (2004) 36-42.

[2] J.L. Anderson, R.L. Taylor Jr., E.C. Smith, W.K. Thomas, S.C. Smith, Differentially expressed genes in aortic smooth muscle cells from atherosclerosissusceptible and atherosclerosis-resistant pigeons, Poult. Sci. 91 (2012) 13151325.

[3] S.J. Assinder, J.L. Stanton, P.D. Prasad, Transgelin: an actin-binding protein and tumor suppressor, Int. J. Biochem. Cell Biol. 41 (2009) 482-486.

[4] D.L. Berlinsky, W. King V, R.G. Hodson, C.V. Sullivan, Hormone induced spawning of summer flounder Paralichthys dentatus, J. World Aquacult. Soc. 28 (1997) 79-86.

[5] J. Bobe, G. Maugars, T. Nguyen, H. Rime, B. Jalabert, Rainbow trout follicular maturational competence acquisition is associated with an increased expression of follicle stimulating hormone receptor and insulin-like growth factor 2 messenger RNAs, Mol. Reprod. Dev. 66 (2003) 46-53.

[6] J. Bobe, T. Nguyen, B. Jalabert, Targeted gene expression profiling in the rainbow trout (Oncorhynchus mykiss) ovary during maturational competence acquisition and oocyte maturation, Biol. Reprod. 71 (2004) 73-82.

[7] J. Bobe, J. Montfort, T. Nguyen, A. Fostier, Identification of new participants in the rainbow trout (Oncorhynchus mykiss) oocyte maturation and ovulationprocesses using cDNA microarrays, Reprod. Biol. Endocrinol. 4 (2006) 39-54.

[8] J. Bobe, T. Nguyen, A. Fostier, Ovarian function of the trout preovulatory ovary: new insights from recent gene expression studies, Comp. Biochem. Physiol. Part A 153 (2009) 63-68.

[9] E. Bonnet, A. Fostier, J. Bobe, Microarray-based analysis of fish egg quality after natural or controlled ovulation, BMC Genom. 8 (2007) 55-71.

[10] B. Campbell, J. Dickey, B. Beckman, G. Young, A. Pierce, H. Fukada, P. Swanson, Previtellogenic oocyte growth in salmon: relationships among body growth, plasma insulin-like growth factor-1, estradiol-17beta, follicle-stimulating hormone and expression of ovarian genes for insulin-like growth factors, steroidogenic-acute regulatory protein and receptors for gonadotropins, growth hormone, and somatolactin, Biol. Reprod. 75 (2006) 34-44.

[11] J. Cerdá, G. Reich, R.A. Wallace, K. Selman, Serotonin inhibition of steroidinduced meiotic maturation in the teleost Fundulus heteroclitus: role of cyclic AMP and protein kinases, Mol. Reprod. Dev. 49 (1998) 333-341.

[12] S. Conner, D. Leaf, G. Wessel, Members of the SNARE hypothesis are associated with cortical granule exocytosis in the sea urchin egg, Mol. Reprod. Dev. 48 (1997) 106-118.

[13] R. Dahle, L. Taranger, Ø. Karlsen, O.S. Kjesbu, B. Norberg, Gonadal development and associated changes in liver size and sexual steroids during the reproductive cycle of captive male and female Atlantic cod (Gadus morhua L.), Comp. Biochem. Physiol. Part A 136 (2003) 641-653.

[14] G. Deng, D.W. Andrews, R.A. Laursen, Amino acid sequence of a new type of antifreeze protein, from the longhorn sculpin Myoxocephalus octodecimspinosis, FEBS Lett. 402 (1997) 17-20.

[15] G. Deng, R.A. Laursen, Isolation and characterization of an antifreeze protein from the longhorn sculpin, Myoxocephalus octodecimspinosis, Biochim. Biophys. Acta 1388 (1998) 305-314.
[16] F. Dubé, P. Amireault, Local serotonergic signaling in mammalian follicles, oocytes, and early embryos, Life Sci. 81 (2007) 1627-1637.

[17] N.J. Duncan, M. Rodriguez, O.G.A. De, D. Alok, Y. Zohar, Effects of controlled delivery and acute injections of LHRHa on bullseye puffer fish (Sphoeroides annulatus) spawning, Aquaculture 218 (2003) 625-635.

[18] L.L. Espey, Ovarian contractility and its relationship to ovulation: a review, Biol. Reprod. 19 (1978) 540-551.

[19] G. Feng, H. Tintrup, J. Kirsch, M.C. Nichol, J. Kuhse, H. Betz, J.R. Sanes, Dual requirement for gephyrin in glycine receptor clustering and molybdoenzyme activity, Science 282 (1998) 1321-1324.

[20] J.-M. Fritschy, R.J. Harvey, G. Schwarz, Gephyrin: where do we stand, where do we go?, Trends NeuroSci 31 (2008) 257-264.

[21] A.F. Garber, S.E. Fordham, J.E. Symonds, E.A. Trippel, D.L. Berlinsky, Hormonal induction of ovulation and spermiation in Atlantic cod Gadus morhua, Aquaculture 296 (2009) 179-183.

[22] S.Y. Gauthier, A.J. Scotter, F.-H. Lin, J. Baardsnes, G.L. Fletcher, P.L. Davies, A reevaluation of the role of type IV antifreeze protein, Cryobiology 57 (2008) 292296.

[23] F.W. Goetz, L. McCauley, G.W. Goetz, B. Norberg, Using global genome approaches to address problems in cod mariculture, ICES J. Mar. Sci. 63 (2006) 393-399.

[24] F.W. Goetz, B. Norberg, L.A.R. McCauley, D.B. Iliev, Characterization of the cod (Gadus morhua) steroidogenic acute regulatory protein (StAR) sheds light on StAR gene function in fish, Comp. Biochem. Physiol. Part B 137 (2004) 351362 .

[25] J.R. Henderson, T. Macalma, D. Brown, J.A. Richardson, E.N. Olson, M.C Beckerle, The LIM protein CRP1 is a smooth muscle marker, Dev. Dyn. 214 (1999) 229-238

[26] M. Hubank, D.G. Schatz, Identifying differences in mRNA expression by representational difference analysis of cDNA, Nucleic Acids Res. 22 (1994) 5640-5648.

[27] G.L. Humason, Animal Tissue Techniques, third ed., W.H. Freeman and Company, San Francisco, 1972.

[28] L. Ibarra-Castro, N.J. Duncan, GnRHa-induced spawning of wild-caught spotted rose snapper Lutjanus guttatus, Aquaculture 272 (2007) 737-746.

[29] K. Iwahashi, T. Kuji, H. Fujiwara, J. Tanaka, N. Takahashi, S. Inagaki, A. Komatsu, Y. Yamamoto, K. Yoshimura, Akagawa, expression of the exocytotic protein syntaxin in mouse oocytes, Reproduction 126 (2003) 73-81.

[30] B. Jalabert, D. Szöllösi, In vitro ovulation of trout oocytes: effect of prostaglandins on smooth muscle-like cells of the theca, Prostaglandins 9 (1975) 765-778

[31] R.S. Kumar, S. Ijiri, J.M. Trant, Molecular biology of the channel catfish gonadotropin receptors: 2 . complementary cDNA cloning, functional expression, and seasonal gene expression of the follicle-stimulating hormone receptor, Biol. Reprod. 65 (2001) 710-717.

[32] H.F. Kwok, W.K. So, Y. Wang, W. Ge, Zebrafish gonadotropins and their receptors. I. Cloning and characterization of zebrafish follicle-stimulating hormone and luteinizing hormone receptors - evidence for their distinct functions in follicle development, Biol. Reprod. 72 (2005) 1370-1381.

[33] W. KingV, P. Thomas, R.M. Harrell, R.G. Hodson, C.V. Sullivan, Plasma levels of gonadal steroids during final oocyte maturation of striped bass, Morone saxatilis L., Gen. Comp. Endocrinol. 95 (1994) 178-191.

[34] O.S. Kjesbu, H. Kryvi, Oogenesis in cod, Gadus morhua L., studied by light and electron microscopy, J. Fish Biol. 34 (1989) 735-746.

[35] C. Ko, C. Gieske, L. Al-Alem, Y. Hahn, W. Su, M.C. Gong, M. Iglarz, Y. Koo, Endothelin-2 in ovarian follicle rupture, Endocrinology 147 (2006) 17701779 .

[36] Y. Kobayashi, T. Kobayashi, M. Nakamura, T. Sunobe, C.E. Morrey, N. Suzuki, Y. Nagahama, Characterization of two types of cytochrome P450 aromatase in the serial sex-changing gobiid fish, Trimma okinawae, Zool. Sci. 21 (2004) 417425.

[37] L. Lanfumey, M. Hamon, $5 \mathrm{HT}_{1}$ receptors, Curr. Drug Targets CNS Neurol. Disord. 3 (2004) 1-10.

[38] D.M. Langenau, F.W. Goetz, S.B. Roberts, The upregulation of messenger ribonucleic acids during 17 $\alpha, 20 \beta$-dihydroxy-4-pregnen-3-one-induced ovulation in the perch ovary, J. Mol. Endocrinol. 23 (1999) 137-152.

[39] J.K. Lee, Y.J. Kim, K.S. Park, S.C. Shin, H.J. Kim, Y.H. Song, H. Park, Molecular and comparative analyses of type IV antifreeze proteins (AFPIVs) from two Antarctic fishes Pleuragramma antarcticum and Notothenia coriiceps, Comp. Biochem. Physiol. Part B 159 (2011) 197-205.

[40] N. Lisitsyn, N. Lisitsyn, M. Wigler, Cloning the differences between two complex genomes, Science 259 (1993) 946-951.

[41] J.-X. Liu, Y.-H. Zhai, J.F. Gui, Molecular characterization and expression pattern of AFPIV during embryogenesis in gibel carp (Carassiu auratus gibelio), Mol. Reprod. Dev. 36 (2009) 2011-2018.

[42] E. Lubenz, G. Young, J. Bobe, J. Cerdá, Oogenesis in teleosts: how fish eggs are formed, Gen. Comp. Endocrinol. 165 (2010) 367-389.

[43] J.A. Luckenbach, F.W. Goetz, P. Swanson, Expressed sequence tags (ESTs) of follicle/interstitial cell enriched ovarian tissue from previtellogenic coho salmon, Cybium 32 (2008) S142-S144.

[44] J.A. Luckenbach, D.B. Iliev, F.W. Goetz, P. Swanson, Identification of differentially expressed ovarian genes during primary and early secondary oocyte growth in coho salmon, Oncorhynchus kitsutch, Reprod. Biol. Endocrinol. 6 (2008) 2-16.

[45] G. Marino, E. Panini, A. Longobardi, A. Mandich, M.G. Finoia, Y. Zohar, C.C. Mylonas, Mylonas, Induction of ovulation in captive-reared dusky grouper 
Epinephelus marginatus (Lowe, 1834), with a sustained-release GnRHa implant, Aquaculture 219 (2003) 841-858.

[46] M. Matsumoto, S. Beltaifa, C.S. Weickert, M.M. Herman, T.M. Hyde, R.C. Saunders, B.K. Lipska, D.H. Weinberger, J.E. Kleinman, A conserved mRNA expression profile of SREB2 (GPR85) in adult human, monkey, and rat forebrain, Mol. Brain Res. 138 (2005) 58-69.

[47] M. Matsumoto, T. Saito, J. Takasaki, M. Kamohara, T. Sugimoto, M. Kobayashi, M. Tadokoro, S. Matsumoto, T. Ohishi, K. Furuichi, An evolutionarily conserved G-protein coupled receptor family, SREB, expressed in the central nervous system, Biochem. Biophys. Res. Commun. 272 (2000) 576-582.

[48] M. Matsumoto, R.E. Straub, S. Marenco, K.K. Nicodemus, S. Matsumoto, A. Fujikawa, S. Miyoshi, M. Shobo, S. Takahashi, J. Yarimizu, M. Yuri, M. Hiramoto, S. Morita, H. Yokoda, T. Sasayama, K. Terai, M. Yoshino, A. Miyake, J.H. Callicott M.F. Egan, A. Meyer-Lindenberg, L. Kempf, R. Honea, R.K. Vakkalanka, J. Takasaki, M. Kamohara, T. Soga, H. Hiyama, H. Ishii, A. Matsuo, S. Nishimura, N. Matsuoka, M. Kobori, H. Matsushime, M. Katoh, K. Furuichi, D.R. Weinberger The evolutionarily conserved $G$ protein-coupled receptor SREB2/GPR85 influences brain size, behavior, and vulnerability to schizophrenia, Proc. Natl. Acad. Sci. USA 105 (2008) 6133-6138.

[49] C. Mittelholzer, E. Andersson, D. Consten, T. Hirai, Y. Nagahama, B. Norberg, 20ß-hydroxysteroid dehydrogenase and CYP19A1 are differentially expressed during maturation in Atlantic cod (Gadus morhua), J. Mol. Endocrinol. 39 (2007) 319-328.

[50] C. Mittelholzer, E. Andersson, G.L. Taranger, D. Consten, T. Hirai, B. Senthilkumaran, Y. Nagahama, B. Norberg, Molecular characterization and quantification of the gonadotropin receptors FSH-R and LH-R from Atlantic cod (Gadus morhua), Gen. Comp. Endocrinol. 160 (2009) 47-58.

[51] C. Mittelholzer, E. Andersson, G.L. Taranger, Ø. Karlsen, B. Norberg, Quantification of gonadotropin subunits $\mathrm{GP} \alpha, \mathrm{FSH} \beta$, and $\mathrm{LH} \beta$ mRNA expression from Atlantic cod (Gadus morhua) throughout a reproductive cycle, Comp. Biochem. Physiol. Part B 153 (2009) 288-295.

[52] C.M. Morrison, Histology of the Atlantic cod, Gadus morhua: An Atlas. Part three: reproductive tract, Can. Spec. Publ. Fish. Aquat. Sci. 110 (1990) 20.

[53] K.Y. Miyasaka, Y.S. Kida, T. Sato, M. Minami, T. Ogura, Csrp1 regulates dynamic cell movements of the mesendoderm and cardiac mesoderm through interactions with dishevelled and diversin, Proc. Natl. Acad. Sci. USA 104 (2007) 11274-11279.

[54] Y. Nagahama, M. Yamashita, Regulation of oocyte maturation in fish, Dev. Growth Differ. 50 (2008) S195-S219.

[55] B.F. O'Dowd, T. Nguyen, A. Marchese, R. Cheng, K.R. Lynch, H.H.Q. Heng, L.F. Kolakowski Jr., S.R. George, Discovery of three novel G-protein-coupled receptor genes, Genomics 47 (1998) 310-313.

[56] K.G. Oikonomou, K. Zachou, G.N. Dalekos, Alpha-actinin: a multidisciplinary protein with important role in B-cell driven autoimmunity, Autoimmun. Rev. 10 (2011) 389-396.

[57] Y. Oka, Y. Sato, H. Tsuda, K. Hanaoka, Y. Hirai, Y. Takahashi, Epimorphin acts extracellularly to promote sorting and aggregation during the condensation of vertebral cartilage, Dev. Biol. 291 (2006) 25-37.
[58] K. Pastorian, L. Hawell III, C.V. Byus, Optimization of cDNA representational difference analysis for the identification of differentially expressed mRNAs, Anal. Biochem. 283 (2000) 89-93.

[59] R. Patiño, P. Thomas, G. Yoshizaki, Ovarian follicle maturation and ovulation: an integrated perspective, Fish Physiol. Biochem. 28 (2003) 305-308.

[60] D.C. Radisky, Y. Hirai, M.J. Bissell, Delivering the message: epimorphin and mammary epithelial morphogenesis, Trends. Cell Biol. 13 (2003) 426-434.

[61] R.K. Ramachandra, S.E. Lankford, G.M. Weber, C.E. Rexroad III, J. Yao, Identification of OORP-T, a novel oocyte-specific gene encoding a protein with a conserved oxysterol binding protein domain in rainbow trout, Mol. Reprod. Dev. 74 (2007) 502-511.

[62] D.V. Rebrikov, O.L. Britanova, N.G. Gurskaya, K.A. Lukyanov, V.S. Tarabykin, S.A Lukyanov, Mirror orientation selection (MOS): a method for eliminating false positive clones from libraries generated by suppression subtractive hybridization, Nucleic Acids Res. 28 (2000) 90-94.

[63] A. Rocha, S. Zanuy, M. Carrillo, A. Gómez, Seasonal changes in gonadal expression of gonadotropin receptors, steroidogenic acute regulatory protein and steroidogenic enzymes in the European sea bass, Gen. Comp. Endocrinol. 162 (2009) 265-275.

[64] P.C. Schroeder, P. Talbot, Ovulation in the animal kingdom: a review with emphasis on the role of contractile processes, Gamete Res. 11 (1985) 191-221.

[65] Z.H. Shehedeh, C.-M. Kuo, K.K. Milisen, Validation of an in vivo method for monitoring ovarian development in the grey mullet (Mugil cephalus L.), J. Fish Biol. 5 (1973) 479-487.

[66] E.R. Simpson, C. Clyne, G. Rubin, W.C. Boon, K. Robertson, K. Britt, C. Speed, M Jones, Aromatase - a brief overview, Annu. Rev. Physiol. 64 (2002) 93-127.

[67] J.L. Song, G.M. Wessel, How to make an egg: transcriptional regulation in oocytes, Differ. 73 (2005) 1-17.

[68] B. Star, A. Nederbragt, S. Jentoft, U. Grimholt, M. Malstrøm, T.F. Gregers, T.B. Rounge, J. Paulsen, M.H. Solbakken, A. Sharma, O.F. Wetten, A. Lanzén, R. Winer, J. Knight, J.-H. Vogel, B. Aken, Ø. Andersen, K. Lagesen, A. ToomingKlunderud, R.B. Edvardsen, K.G. Tina, M. Espelund, C. Nepal, C. Previti, B.O Karlsen, T. Moum, M. Skage, P.R. Berg, T. Gjøen, H. Kuhl, J. Thorsen, K. Malde, R. Reinhardt, L. Du, S.D. Johansen, S. Searle, S. Lien, F. Nilsen, I. Jonassen, S.W. Omholt, N.C. Stenseth, K.S. Jakobsen, The genome sequence of Atlantic cod reveals a unique immune system, Nature 477 (2011) 207-210.

[69] P.F. Valerio, S.V. Goddard, M.H. Kao, G.L. Fletcher, Survival of northern Atlantic cod (Gadus morhua) eggs and larvae when exposed to ice and low temperature, Can. J. Fish. Aquat. Sci. 49 (1992) 2588-2595.

[70] D.L. Villeneuve, N. Garcia-Reyero, D. Martinović, J.E. Cavallin, N.D. Mueller, L.C. Wehmas, M.D. Kahl, A.L. Linnum, E.J. Perkins, G.T. Ankley, Influence of ovarian stage on transcript profiles in fathead minnow (Pimephales promelas) ovary tissue, Aquat. Toxicol. 98 (2010) 354-366.

[71] R.A. Wallace, K. Selman, Ultrastructural aspects of oogenesis and oocyte growth in fish and amphibians, J. Electron Microsc. Tech. 16 (1990) 175-201.

[72] Y. Zohar, C.C. Mylonas, Endocrine manipulations of spawning in cultured fish: from hormones to genes, Aquaculture 197 (2001) 99-136. 